\title{
Ultrastructural and Microanalytical Study of a Subcutaneous Granuloma HPV Tetravalent Vaccine Induced: A Case Report and Review of Literature
}

\author{
Manuel Scimeca ${ }^{\mathrm{a}}$, Lucia Anemona ${ }^{\mathrm{a}}$, Alfredo Colantoni ${ }^{\mathrm{b}}$, Elena Bonanno ${ }^{\mathrm{a}, \mathrm{c}}$
}

\begin{abstract}
We report the case of an eighteen years old woman who developed a subcutaneous nodular lesion localized on her right upper arm. Conventional histological examination revealed a lesion characterized by a necrotic center surrounded by palisading epithelioid histiocytes embedded into the fibroadipous and skeletal muscle tissue. Transmission electron microscopy analysis confirmed the histological observations in terms of cellular composition of the granuloma. The ultrastructural microanalysis shed light on the elemental composition of the examined granulomatous formation since it clearly detected metallic deposits with an emission pick at EDX for aluminum within the hystocites's granules. Our results support previous published data on the reactive nature of granulomatous reaction at the site of vaccine. The presence of aluminum inside the cytoplasm of macrophages proved by microanalysis gives strength to the etiopatogenic hypothesis of the granuloma formation.
\end{abstract}

Keywords: Granuloma; HPV vaccine; Transmission electron microscopy; EDX Microanalysis; Aluminum

\section{Introduction}

HPV infections are the most common sexually transmitted infections in the United States and account 5 percents of all cancers worldwide [1]. More than half of sexually active people are infected with one or more HPV types at some point in their lives. Recent research indicates that 42.5 per-

\footnotetext{
Manuscript accepted for publication March 20, 2013

${ }^{a}$ Department of Biomedicine and Prevention, University of Rome "Tor Vergata", Via Montpellier 1, Rome 00133, Italy

bepartment of Systems Medicine, University of Rome "Tor Vergata", Via Montpellier 1, Rome 00133, Italy

${ }^{\mathrm{c}}$ Corresponding author: Elena Bonanno, Department of Biomedicine and Prevention, University of Rome "Tor Vergata", Via Montpellier 1, Rome 00133, Italy. Email: elena.bonanno@uniroma2.it
}

doi: http://dx.doi.org/10.4021/jmc1193w cent of women have genital HPV infections, whereas less than 7 percent of adults have oral HPV infections [2,3]. Potentially, all cervical cancers are caused by HPV infections, with just two HPV types, 16 and 18, responsible for about 70 percent of all cases [4, 5]. In 2006 a tetravalent human papillomavirus (HPV) vaccine was licensed for use in women, aged 9 - 26 years [6]. The L1 major capsid protein of HPV is the antigen used for HPV vaccination [7]. Recombinant DNA technology allows the production of the L1 protein in Saccharomyces cerevisiae so that protein can self-assemble into not infective virus-like particles (VLP). VLP was adsorbed on an aluminum-coating adjuvant [2]. Adverse events associated to the administration of the HPV tetravalent vaccine include pain, swelling and erythema, whereas hives, gastroenteritis, headache/hypertension, vaginal hemorrhage and broncospasm are rare [8]. Among the reported side effects there is no reference about the possibility of the development of a granulomatous reaction.

In this report, we describe the study of a granuloma arisen in a patient after the administration of the HPV tetravalent vaccine. The granuloma has been evaluated by histology, electron microscopy and energy-dispersive x-ray (EDX) microanalysis. In particular, EDX microanalysis, used to investigate the elemental composition of the histiocytes's granules within the analyzed granuloma, allowed us to demonstrate the presence of aluminum inside hystiocytes present in the lesion.

\section{Case Report}

We report the case of an eighteen years old girl who developed a subcutaneous nodular lesion localized on her right upper arm. The lesion was excised by surgical resection and submitted to histological analysis. Macroscopic examination revealed a soft subcutaneous nodule, whitish-gray, well circumscribed, measuring $1 \times 2 \mathrm{~cm}$. A thin slice of the lesion was fixed in Karnowsky solution and embedded in resin for ultrastructural analysis and investigations by microanalysis technique.

Conventional histological examination revealed a lesion characterized by a necrotic center surrounded by palisading 


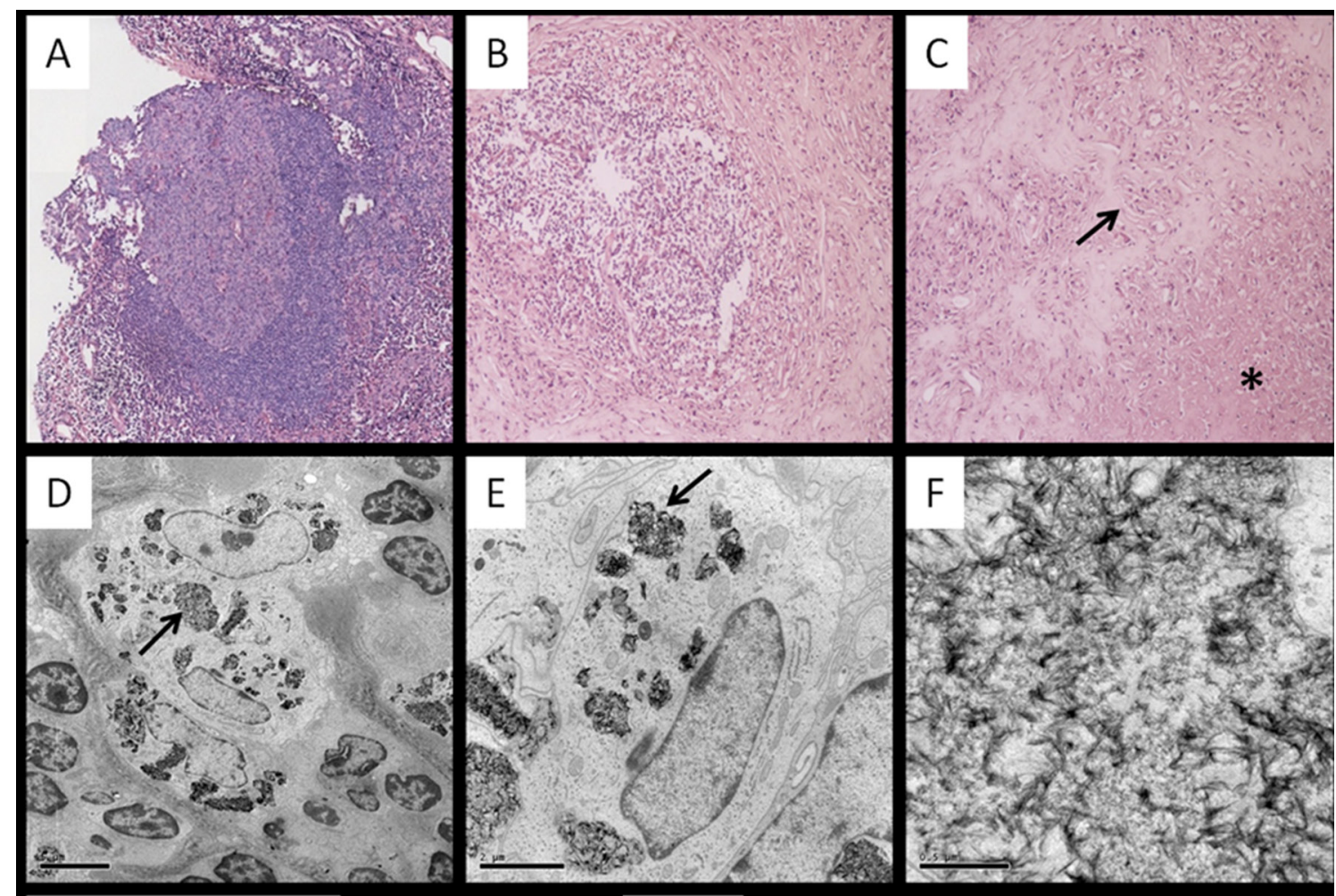

Full scale counts: 125

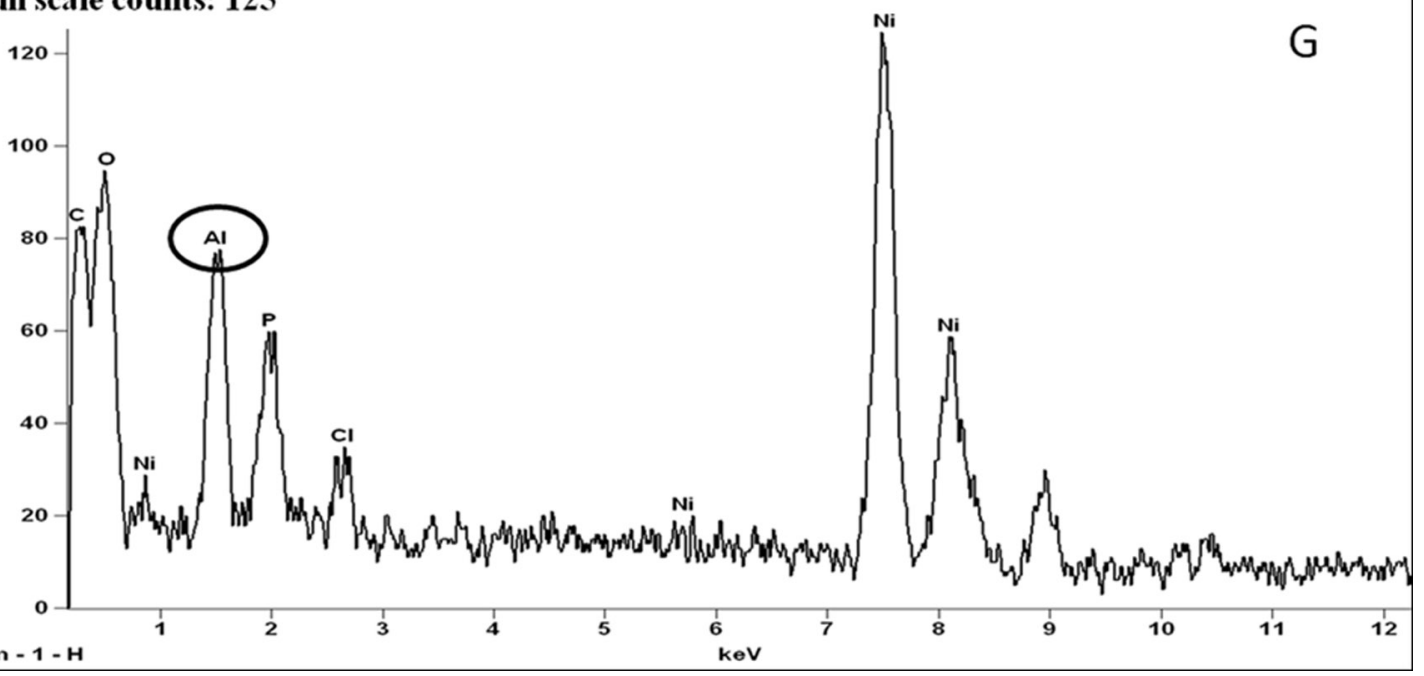

Figure 1. panel $A$ to $C$ : hematoxylin eosin section of the lesion showing a granulomatous reaction with lymphatic follicle formation (panel A magnification $4 \times$ ), macrophages and lymphoplasmacellular infiltrate (panel B, MAGNIFICATION $10 \times$ ). The lesion is characterized by a necrotic center (asterisk in panel C) surrounded by palisading epithelioid histiocytes (arrow in panel C). PANEL D to F: conventional transmission electron microscopy characterization of the lesion. PANEL D shows a big histiocyte's granule (arrow) containing fibrillar electron dense material (MAGNIFICATION 3,500 $\times$ ). The ultrastructural study demonstrated the presence of needle-shaped crystalline material with variable thickness (PANEL E 10,000 $\times$-arrow-; PANEL F 50,000 ×). EDX microanalysis spectrum (PANEL G) reveals the presence of large amounts of aluminum (circle) and of elements referable to the nickel support (Ni) and to biological compounds (P, CL, C and O).

epithelioid histiocytes embedded into the fibroadipous and skeletal muscle tissue (Fig.1 panel A, B and C). This granulomatous lesion closely resembled a deep granuloma annulare or rheumatoid nodule. The histiocytes contained abun- dant intracytoplasmic violaceous/gray granular material PAS and Ziehl-Nielsen negative, excluding the presence of fungi and of alcohol acid resistant microorganisms.

The inflammatory cells of the granuloma were CD 20 
and CD 3 positive, CD 15 and CD 30 negative; a mild positivity for CD 68 could be detected. These data and the follicles-like organization of the lesion, pointing out an inflammatory infiltrate, rich in B and T lymphocytes, excluded the diagnosis of lymphoma.

Transmission electron microscopy analysis (Fig.1 panel $\mathrm{D}, \mathrm{E}$ and $\mathrm{F}$ ) confirmed the histological observations in terms of cellular composition of the granuloma. Higher magnifications revealed the presence of abundant histyocites's granules containing a needle-shaped, crystalline material with variable thickness.

The ultrastructural microanalysis shed light on the elemental composition of the examined granulomatous formation since it clearly detected metallic deposits with an emission pick at EDX for aluminum within the hystocites's granules. The obtained spectrums revealed the presence of large amounts of aluminum, elements referable to the nickel support $(\mathrm{Ni})$ and to biological compounds $(\mathrm{C}, \mathrm{Cl}, \mathrm{P}$ and $\mathrm{O})$ (Fig.1 panel G).

Anamnestic data revealed that the patient was negative for nodal and spleen enlargement and for any recent infectious disease or beetle bite near the site of the lesion. The only relevant data were that she approximately one year before the onset of the granuloma was submitted to tetravalent HPV-vaccine inoculums at the site of the granulomatous lesion.

It is interesting to note that the adjuvant system of the tetravalent HPV is made of aluminum salts. Other anamnestic data were not contributory. Therefore the histological and ultrastructural reported data were consistent with the diagnosis of subcutaneous granuloma related to the aluminum present in the vaccine inoculum. The patient recovered well from her procedure and had no further complications (two years followup).

\section{Histological analysis}

After fixation in $10 \%$ buffered formalin for 24 hours, the tissue was embedded in paraffin, sectioned and stained with hematoxylin-eosin [9].

For immunohistochemistry, $4 \mu \mathrm{m}$ paraffin sections have been processed by the automatized system Bench Mark Ventana (F. Hoffmann-La Roche AG, Konzern-Hauptsitz Grenzacherstrasse 124 CH-4070 Basel, Schweiz). Sections, after pretreatment, were incubated with mouse anti-CD20 (clone L26, Roche) rabbit anti-CD3 (clone 2GV6, Roche), rabbit anti-CD15 (clone MMA, Roche) and mouse anti-CD68 (clone HP-1, Roche). The reactions were revealed by nonbiotin peroxidase detection system Ventana (F. Hoffmann-La Roche AG, Konzern-Hauptsitz Grenzacherstrasse $124 \mathrm{CH}-$ 4070 Basel, Schweiz).

PAS and Ziehl-Nielsen stainings have been performed by Artisan automatized system (Dako Denmark A/S Produktionsvej 42 Glostrup Denmark).

\section{Transmission electron microscopy and microanalysis}

A section of the sample was immediately fixed by immersion in Karnovsky solution [10] and then post-fixed in Osmium Tetroxide $2 \%$ [11]. After washing with phosphate buffer 0.1 $\mathrm{M}$, the sample was dehydrated by a series of incubations in $30 \%, 50 \%, 70 \%, 95 \%$ ethanol. Dehydration has been continued by incubations in absolute ethanol and propylene oxide. The sample was then embedded in Epon 812 [12] (Agar Scientific Elektron Technology UK Ltd Unit 7, Stansted, Essex CM24 8GF United Kingdom).

After inclusion the tissue was cut $[13,14]$ and stained with heavy metals solutions (uranium acetate and lead citrate) as described by Reynolds [15].

Ultrathin-sections, approximately $100 \mathrm{~nm}$ thick, were mounted on aluminum grids for microanalysis. EDX spectra of hystocites's granules in non-stained sections were acquired on a Hitachi 7100FA transmission Electron Microscope (AEM) equipped with a Gatan BioScan Camera model 820 and a Thermo Scientific EDX detector at an acceleration voltage of $75 \mathrm{KeV}$ and magnification of 12000 . Spectra were semi-quantitatively analyzed by the Noram System Six software using the standardless Cliff-Lorimer k-factor method [16].

To calibrate the EDX apparatus reference standard for x-ray microanalysis of MAC industry (Micro-Analysis Consultants Ltd, 19 Edison Road, St.Ives Industrial Estate, St.Ives, Cambridgeshire PE27 3LF UK) has been used.

\section{Discussion}

In this paper we report, for the first time, a transmission electron microscopy analysis associated to the elemental composition study in order to demonstrate the presence of aluminum inside the cells of a granulomatous reaction at the inoculum site of HPV vaccine.

Recently, a granulomatous reaction related to aluminum HPV vaccine has been described by Marsee DK. et al. [17]. This evidence was demonstrated through the histochemical staining "alluminon" (aurintricarboxylate aluminum).

Our data confirm and give strength to this observation since the "alluminon" method used by Marsee resulted to be not so specific, due to the simultaneous reaction with aluminum and other elements, such as iron and beryllium. The intensity of the staining obtained by "alluminon" method is subjected to the presence of interfering ions. Stain formation, which occurs only over a limited range of $\mathrm{Al}$ concentrations, is prevented by the presence of some chemical agents [18].

Ultrastructural microanalysis provided a punctual analysis of the tissue with a high specificity, distinguishing among the different chemical elemental forms and associating the microanalitical data to the ultrastuctural morphology.

This advantageous technique allows us to exclusively 
analyze the inside of the hystiocites's granules, determining the elemental composition of the resident crystals. The specificity of the analysis is evaluated through the parallel analysis of the MAC's sample standards.

A wider case study about the formation of persistent nodules at the injection sites of not HPV vaccines has been reported. Bergfors et al. [19] described the formation, in 19 children, of persistent pruritic nodules at the injection sites of vaccines adsorbed with aluminum's salts, most commonly reported after diphtheria-tethanus, pertussis and B-hepatitis vaccines.

Epicutaneous testing for delayed hypersensitivity to aluminum had been used to demonstrate the immune reaction against this metal. Any histological study was added to this test.

Choget et al. [20] described 14 cases of patients different for sex and age who developed aluminum granulomas at the vaccine injection sites, correlated to histological data. The morphological analysis of these samples revealed different histological patterns such as panniculitis reaction with or without foreign body-type giant cells, pseudo-lymphoma and necrotizing granuloma. Many times the characterization of histiocyte's granules becomes essential for the differential diagnosis. Exclusion of malignant pathology (i.e. lympho$\mathrm{ma}$ ) in the differential diagnosis underlies the importance of a fine morphological characterization of the lesion.

To this end, we consider notable the work of Morroni et al. [21], that in 1995 studied a subcutaneous granuloma through EDX microanalysis associated to the scanning electron microscopy and the ESI (electron spectroscopic imaging) technology.

In our study, thanks to the EDX apparatus, directly associated to the electron transmission microscope, we could find a fine correspondence between the ultrastuctural datum and the microanalitical one.

In conclusion, our results support previous published data about the reactive nature of granulomas at the site of vaccine. The presence of aluminum inside the cytoplasm of macrophages, proved by microanalysis, gives strength to the etiopathogenic hypothesis of the granuloma formation. Moreover these data suggest a specific role of the ultrastructural and microanalitical analysis in the clinical practice with regard to the differential diagnosis of metal/pollutant related pathology.

\section{References}

1. Parkin DM. The global health burden of infectionassociated cancers in the year 2002. Int J Cancer. 2006;118(12):3030-3044.

2. Hariri S, Unger ER, Sternberg M, Dunne EF, Swan D, Patel S, Markowitz LE. Prevalence of genital human papillomavirus among females in the United States, the
National Health And Nutrition Examination Survey, 2003-2006. J Infect Dis. 2011;204(4):566-573.

3. Gillison ML, Broutian T, Pickard RK, Tong ZY, Xiao W, Kahle L, Graubard BI, et al. Prevalence of oral HPV infection in the United States, 2009-2010. JAMA. 2012;307(7):693-703.

4. Schiffman M, Castle PE, Jeronimo J, Rodriguez AC, Wacholder S. Human papillomavirus and cervical cancer. Lancet. 2007;370(9590):890-907.

5. Munoz N, Bosch FX, Castellsague X, Diaz M, de Sanjose S, Hammouda D, Shah KV, et al. Against which human papillomavirus types shall we vaccinate and screen? The international perspective. Int $\mathrm{J}$ Cancer. 2004;111(2):278-285.

6. Markowitz LE, Dunne EF, Saraiya M, Lawson HW, Chesson H, Unger ER. Quadrivalent Human Papillomavirus Vaccine: Recommendations of the Advisory Committee on Immunization Practices (ACIP). MMWR Recomm Rep. 2007;56(RR-2):1-24.

7. Stanley M, Lowy DR, Frazer I. Chapter 12: Prophylactic HPV vaccines: underlying mechanisms. Vaccine. 2006;24(Suppl 3):S3/106-113.

8. Food and Drug Administration. Product approval information---licensing action . Gardasil (quadrivalent human papillomavirus types 6,11,16,18). Merck \& Co., Whitehouse Station, NJ. Available at http://www.fda. gov/cber/label/HPVmer060806LB.pdf.

9. Carson FL (1997) Histotechnology: a self-instructional text. Chicago: American Society for Clinical Pathology 1997; 6: 93..

10. Karnovsky MJ (1965) A formaldehyde-glutaraldeyde fixative of high osmolarity for use in electron microscopy., Journal of Cell Biology; 27:137A.

11. Hayat MA (1981) Fixation for electron microscopy. New York: Academic Press.

12. Luft JH. Improvements in epoxy resin embedding methods. J Biophys Biochem Cytol. 1961;9:409-414.

13. Reid N (1975) Ultramicrotomy. In: Glauert A.M., ed. Practical methods in electron microscopy. Amsterdam: North Holland; Vol. 3 Part2.

14. Dykstra MJ (1992) Biological electron microscopy: theory, techniques and troubleshooting. New York: Plenum Press.

15. Reynolds ES (1963) The use of lead citrate at high $\mathrm{pH}$ as an electron opaque stain based on metal chelation. Journal of Cell Biology.

16. Fernandez-Segura E, Warley A. Electron probe X-ray microanalysis for the study of cell physiology. Methods Cell Biol. 2008;88:19-43.

17. Marsee DK, Williams JM, Velazquez EF. Aluminum granuloma after administration of the quadrivalent human papillomavirus vaccine. Report of a case. Am J Dermatopathol. 2008;30(6):622-624.

18. Clark RA, Krueger GL. Aluminon: its limited applica- 
tion as a reagent for the detection of aluminum species. J Histochem Cytochem. 1985;33(7):729-732.

19. Bergfors E, Bjorkelund C, Trollfors B. Nineteen cases of persistent pruritic nodules and contact allergy to aluminium after injection of commonly used aluminiumadsorbed vaccines. Eur J Pediatr. 2005;164(11):691697.

20. Chong H, Brady K, Metze D, Calonje E. Persistent nodules at injection sites (aluminium granuloma) -clinicopathological study of 14 cases with a diverse range of histological reaction patterns. Histopathology. 2006;48(2):182-188.

21. Morroni M, Barbatelli G, Carboni V, Sbarbati A, Cinti S. Subcutaneous nodules in a patient hyposensitized with aluminium-containing allergen extracts: a microanalytical study. Anal Cell Pathol. 1995;9(3):235-241. 